\title{
Betrachtungen einer Millennial über das „Neue Deutsche Problem“ nach 30 Jahren Frieden
}

https://doi.org/10.1515/sirius-2021-3007

Als ich Teenager war, hörte ich gern die deutsche HipHop-Reggae-Band Freundeskreis. In einem ihrer Songs gibt es die Textzeile Geschichte ist ,was, das lange her ist oder immer ohne einen passiert“. Es war Ende der 1990er Jahre, ich saß in meinem Kinderzimmer in der deutschen Vorstadt - und ich weiß noch genau wie richtig sich dieser Satz anfühlte. Die ideologischen Schlachten der Geschichte waren geschlagen, nichts passierte mehr. Ruhig, gemütlich - ein bisschen langweilig. Im Rückblick lächle ich ob meiner naiven Teenagerangst, etwas zu verpassen. Die Geschichte endete nicht, und ich würde gern meinem jüngeren Selbst sagen, dass es nicht unbedingt angenehm ist, in aufregenden Zeiten zu leben. Aber jetzt, da meine Generation in außenpolitische Entscheidungspositionen gelangt, lohnt es sich, darüber nachzudenken, wie unsere Geschichte unser Denken geprägt hat.

Thomas Bagger, deutscher Diplomat und Berater des Bundespräsidenten, sagte einmal: „Das Ende der Geschichte war eine amerikanische Idee, aber eine deutsche Realität. ${ }^{1}$ Ich würde dem hinzufügen: ,,... und ist jetzt ein Problem der Millennials.“ Denn Bagger hat Recht: Das „Ende der Geschichte“ war, bis vor kurzem, eine deutsche Realität - sowohl in dem ideologischen Sinne, in dem es der Vater des Konzepts, der amerikanische Politikwissenschaftler Francis Fukuyama, verstand, als auch in dem vereinfachten Sinne, dass „sehr wenig passiert.“ Dies stellt deutsche Millennials - diejenigen von uns, die in dieser Zeit aufwuchsen - vor eine besondere Herausfor-

Anmerkung: Dieser Beitrag erschien zuerst in englischer Sprache bei War on the Rocks am 19 Mai 2021, Abdruck mit freundlicher Genehmigung von „War on the Rocks.“

1 Man muss der Ehrlichkeit halber hinzufügen, dass Thomas Bagger diesen Satz dem bulgarischen Autor Ivan Krastev zuschreibt, Krastev wiederum behauptet, der Satz käme von Thomas Bagger.

*Kontakt: Dr. Ulrike Franke ist Senior Policy Fellow beim European Council on Foreign Relations. Sie veröffentlicht den Podcast Sicherheitshalber, einen deutschsprachigen Podcast über Sicherheitsund Verteidigungspolitik; E-Mail: Ulrike.Franke@ecfr.eu derung. Deutschen Millennials fällt es schwer, sich auf die Welt einzustellen, in der wir heute leben. Wir denken nicht in Kategorien von Interessen und tun uns schwer mit dem Konzept geopolitischer Macht, und mit der Tatsache, dass militärische Macht ein Element geopolitischer Macht ist. Das ist insofern besorgniserregend, als dass Deutschland eine wichtige Rolle im internationalen System zukommt.

\section{Eine deutsche Herausforderung}

Wir stehen am Beginn einer Phase des geopolitischen Wettbewerbs und möglicher internationaler Instabilität. In diesem Zusammenhang schauen viele auf Deutschland. Deutschland soll dabei helfen, die freiheitliche Weltordnung zu verteidigen. Es soll die Europäische Union zusammenhalten und ihr helfen, zwischen einem aufstrebenden China und den im Niedergang begriffenen Vereinigten Staaten zu navigieren. Vor dem größten und wirtschaftlich stärksten europäischen Land, dessen wirtschaftliches und soziales Wohlergehen vom Welthandel und von internationaler Stabilität abhängen, liegt eine schwere Aufgabe. Dies sind herausfordernde Zeiten - aber es ist nicht das erste Mal, dass ein Land in einem sich wandelnden internationalen Umfeld agieren muss. Tatsächlich gibt es eine Methode, um solche Herausforderungen zu bewältigen: Man definiert seine Interessen und Prioritäten, man macht eine Bestandsaufnahme seiner Fähigkeiten und überlegt, wie man ausreichende Ressourcen zur Erreichung seiner Ziele sicherstellt. Man sucht Wege, um durch Bündnisse, veränderte Finanzierungsprioritäten und anderes mehr Fähigkeiten zu verbessern. Man formuliert eine Strategie, um die Ziele mit diesen Fähigkeiten zu erreichen. Während man dies tut, wendet man die gleiche Methode an, um seine Gegner einzuschätzen. Was sind ihre Interessen? Was wollen sie tun? Wozu sind sie in der Lage? Was können sie vielleicht erreichen? Dies lässt sich nicht mathematisch exakt berechnen - es gibt Unsicherheiten, unvollständige Information und das menschliche Element. Und zweifellos wurde nicht jede politische Entscheidung - von der Westbindung bis zum Krieg gegen Terrorismus - ausschließlich auf Grundlage dieser Methode getroffen. Aber 
sie sollte der Ausgangspunkt jeder außenpolitischen Entscheidung sein. Ein derartiges strategisches Denken hilft, den außenpolitischen Reflexionsprozess zu lenken.

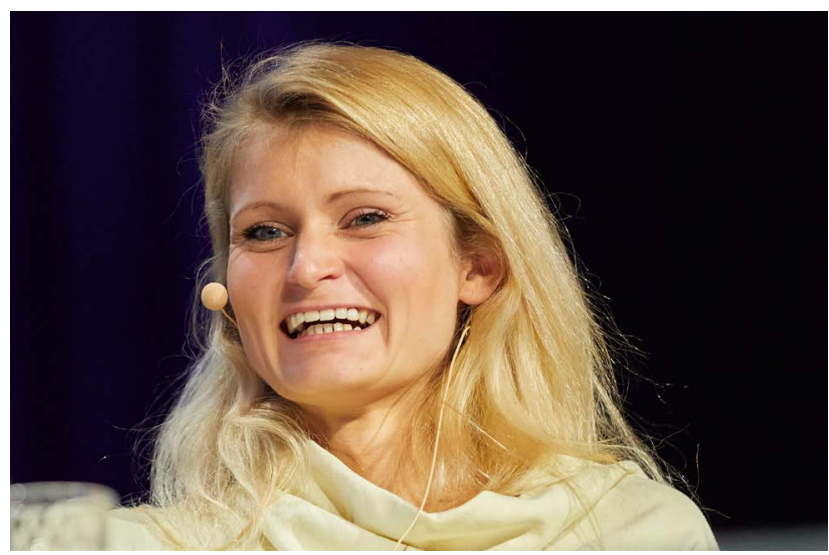

Abb. 1: Ulrike Franke

Leider ist diese Art des strategischen Denkens etwas, was jüngeren deutschen außenpolitischen Entscheidungsträgern nicht leichtfällt. Es ist ihnen fremd. Dreißig Jahre lang lebten wir angenehm abgeschottet von der rauen Welt der Machtpolitik. Die außergewöhnlichen internationalen Rahmenbedingungen, unter denen wir aufgewachsen sind, waren für uns der Normalzustand. Die Ideen, die aus 1989 erwuchsen, waren unsere Überzeugungen. Jetzt, da die Geopolitik und insbesondere die geopolitische Machtpolitik zurück sind, sind wir orientierungslos.

Es hat eine Weile gedauert, bis mir auffiel, dass deutsche Millennials (die ältesten von ihnen wurden Anfang der 1980er Jahre geboren) teils recht ungewöhnliche außenpolitische Vorstellungen haben. Je länger ich außerhalb Deutschlands lebte und insbesondere in Ländern, in denen geopolitisches und strategisches Denken selbstverständlicher ist, umso mehr haben mich einige der Diskussionen verblüfft, die meine Altersgenossen in Deutschland führten. Ein deutscher Millenial brachte es einmal wunderbar auf den Punkt: „Geopolitik hört sich einfach zu sehr nach Truppenbewegung an!“, sagte er mir. Dieser Satz ist Ausdruck einer Reihe expliziter und implizierter Ideen und Überzeugungen, die mir oft bei Gleichaltrigen in Deutschland begegnen: eine Skepsis gegenüber der Geopolitik; der Unwille, in Begriffen von Macht und Interessen zu denken, und die Ablehnung des Militärs als einem Werkzeug der Politik. Deutsche Millennials betrachten die internationale Politik aus einer wertebasierten und von Emotionen geprägten Perspektive, nicht auf der Grundlage von Interessen. Selbstverständlich schließen sich Werte und Interessen nicht gegenseitig aus und sie sind oftmals in einer Weise miteinander verwoben, die es schwermacht sie auseinanderzuhalten. Aber als Deutsche haben wir gelernt, den Interessen-Faktor grundsätzlich abzulehnen. Meine Generation hat eine geradezu romantische Idee der internationalen Beziehungen entwickelt. Wir sehen Allianzen als Freundschaften und Meinungsverschiedenheiten als Ausdruck von Wertedifferenzen. Und deutsche Millennials tun sich schwer mit dem Militär - insbesondere mit der Vorstellung, dass das Militär ein Element geopolitischer Macht ist, das man braucht, um geopolitisch Einfluss zu haben. Diese Einstellung ist generell in der deutschen Bevölkerung weit verbreitet. Aber wie eine aktuelle Umfrage zeigt, ist sie unter Millennials besonders stark ausgeprägt: Die Zustimmung zu einer Kürzung des deutschen Verteidigungshaushalts ist unter Millennials höher als bei jeder anderen Altersgruppe, während die Unterstützung für eine Budgeterhöhung bei den Millennials niedriger ist als in allen anderen Altersgruppen.

Wir haben intellektuell - und praktisch - abgerüstet. Da wir unsere strategischen Muskeln nie trainieren mussten, sind sie verkümmert. Machtpolitik steht in Widerspruch $\mathrm{zu}$ unserem Verständnis internationaler Beziehungen. Wir sind nicht gewohnt, in diesen Kategorien zu denken, und wir sprechen diese Sprache nicht und wir sind daher in keiner Weise vorbereitet, Gegnern mit anders gelagerten Interessen, die immer lautstärker das in Frage stellen, was wir als das einzige System ansehen, entgegenzutreten.

\section{Wie ist es dazu gekommen?}

Checkyour history. Wir alle werden von der Umwelt geprägt, in der wir aufwachsen. Aber während dies in sozioökonomischer Hinsicht allgemein anerkannt ist, haben nur wenige von uns darüber nachgedacht, was dies in (geo-) politischer Hinsicht bedeutet. Man bringt uns bei, unsere Privilegien kritisch zu hinterfragen (check your privilege), aber wie viele Menschen erkunden die zeitgeschichtlichen Prägungen ihrer Einstellungen? Generationen werden oftmals durch bedeutende Ereignisse definiert - sie schweißen eine Generation zusammen, geben ihr ein Thema. Es erzeugt Bezugspunkte, wenn Menschen im gleichen Alter die gleichen einschneidenden Momente durchmachen und die gleichen Umbrüche erleben. Selbstverständlich werden bedeutende Ereignisse niemals nur von einer Generation erlebt, da zu jedem beliebigen Zeitpunkt Menschen aus drei bis fünf Generationen am Leben sind. Aber Bezugspunkte für das, was als Normalität angesehen wird, werden in den ersten Lebensjahrzehnten festgelegt. 
Als Millennials werden all diejenigen bezeichnet, die zwischen Anfang bis Mitte der 1980er und Ende der 1990er Jahre geboren wurden. Wir verdanken unseren Namen der Jahrtausendwende, die wir in jungem Alter erlebten. Aber auch wenn Silvester 1999/2000 ein frohes Fest war, war es doch kein Ereignis von grundlegender Bedeutung. Tatsächlich würde ich sagen, dass meine Generation in Deutschland kein grundlegendes, verbindendes Ereignis erlebt hat. Vielmehr ist der bedeutendste und wirkmächtigste Moment für meine Generation ein Ereignis, an das sich nur wenige von uns auch nur erinnern können, weil wir entweder noch nicht geboren oder nicht alt genug waren, um zu verstehen, was vor sich ging: 1989, der Fall der Berliner Mauer. Er war der Auftakt zum Ende der Sowjetunion, der zur Umwälzung des gesamten geopolitischen Systems führte und den Weg zur globalen Unipolarität ebnete. Für die Deutschen war 1989 das Jahr, in dem sie für lange Zeit zum letzten Mal mit Geopolitik konfrontiert waren. Jetzt, wo meine Alterskohorte in Machtpositionen gelangt, ist es höchste Zeit, dass wir uns unserer Geschichte zuzuwenden und unsere blinden Flecken beseitigen.

An dieser Stelle muss ich eingestehen, dass auch ich einen blinden Fleck habe: Ich spreche von deutschen Millennials, aber ich vermute, dass ostdeutsche Millennials manche Dinge anders sehen. Im Gegensatz zu den westdeutschen Erfahrungen, die ich weiter unten beschreibe - Stabilität und die tiefe Überzeugung, dass ihr System das endgültige ist - wurden Ostdeutsche meiner Generation in eine Welt hineingeboren, die sich in einem Prozess des Zerfalls befand. Die DDR wurde 1990 aufgelöst, es folgte eine vollständige Neuausrichtung der ostdeutschen Wirtschaft und die Einführung einer neuen Währung. Ostdeutschland wurde von einer Wirtschaftskrise erschüttert, und viele Unternehmen gingen pleite, die Arbeitslosigkeit stieg. Die politische Partei - und deren Ideologie -, die jahrzehntelang tonangebend gewesen war, verschwand. Dieser Prozess hielt für diejenigen, die ihn erlebten, zweifellos besondere Lehren bereit, über die ich jedoch nicht berichten kann. Obgleich ich fest davon überzeugt bin, dass sich meine Erfahrungen und die daraus gezogenen Lehren bis $\mathrm{zu}$ einem gewissen Grad auf andere Europäer und die westliche Welt generell verallgemeinern lassen, trifft, was ich beschreibe, wahrscheinlich am besten auf die westdeutsche, europhil gebildete Mittelschicht zu. Und auch wenn das nicht perfekt repräsentativ sein mag, so beschreibt es nun einmal viele der Millennials, die gegenwärtig in der politischen Welt ihren Weg machen.

Deutsche Millennials sind aus zwei Gründen nicht gewappnet für eine Welt, in der strategisches Denken von
Bedeutung ist. Erstens wuchsen wir in einer Zeit außergewöhnlicher geopolitischer Stabilität auf. Genau das bringt das Freundeskreis Lied zum Ausdruck: Wir hatten nie das Gefühl, Teil des permanenten historischen Wandlungsprozesses zu sein, vielmehr hatten wir den Eindruck, außerhalb davon zu stehen, im Nachhinein geboren zu sein. Das Bestreben, Politik zu verstehen, erschien uns genauso wichtig wie der Erwerb geografischer, geometrischer oder geologischer Kenntnisse - alles interessante Fachgebiete, aber ohne direkten Bezug zu unserem Leben.

Zweitens, niemand auf der Welt hat die Idee vom „Ende der Geschichte“ so enthusiastisch angenommen und verinnerlicht wie die Deutschen. Deutsche, die das Jahr 1989 erlebt hatten, machten sich die Idee zu eigen, dass ideologischer Wettstreit eine Sache der Vergangenheit sei - und deutsche Millennials verinnerlichten dies als eine historische Wahrheit. Die Lösung für politische Diskussionen war von denjenigen gefunden worden, die vor uns kamen, und das beste System hatte sich durchgesetzt - vielleicht könnten wir im Bereich der Sozialpolitik ein paar Fortschritte machen, aber im Übrigen könnten wir uns anderen Dingen zuwenden.

\section{Ein ruhiger Normalzustand}

Jeder Deutsche, der zu jung ist, um sich an das Ende der Sowjetunion und die deutsche Wiedervereinigung zu erinnern, ist in einer Welt außergewöhnlicher Stabilität und Frieden aufgewachsen. Militärisch wurden wir von den USA und der NATO beschützt, und daher mussten wir nie über die Rolle des Militärs nachdenken. Das war für meine Generation natürlich großartig. Aber es hatte weitreichende Auswirkungen darauf, wie wir die Welt sehen und was wir für normal halten.

Deutschland stand oft im Mittelpunkt der europäischen und der Weltpolitik. Die deutsche Geschichte war eine Achterbahn sich verändernder Grenzen und politischer Organisationsformen, ideologischer Kämpfe, Kriege und Konflikte gewesen. Aber nach 1989 und der deutschen Wiedervereinigung im Jahr 1990 beruhigte sich die Lage ganz erheblich. Auf Deutschland traf Francis Fukuyamas berühmtes Konzept vom „Ende der Geschichte“ sogar in seiner einfachsten Auslegung zu: Seit 1989 ist in Deutschland wenig passiert.

Selbstverständlich stand die Welt während der letzten 30 Jahre nicht völlig still. Aber die Anschläge vom 11. September 2001, der Krieg gegen den Terrorismus, oder die Finanzkrise waren alles keine Ereignisse, die direkt uns widerfuhren. Die Bundeswehr zog in Afghanistan in den 
Krieg, aber dies wirkte sich nicht auf die Gesellschaft zuhause aus. Die Invasion des Irak in 2003 ließ einige Millennials gegen amerikanischen Imperialismus demonstrieren, aber im Übrigen war sie weit von unserem Alltag entfernt. Die weltweiten Konflikte schienen die Tatsache zu bezeugen, dass andere noch nicht eingesehen hatten, dass ideologische Kämpfe sinnlos waren. Die Finanzkrise kam einem definierenden Ereignis für deutsche Millennials vielleicht am nächsten, aber da Deutschland gut durch die Krise kam, verstärkte dies nur den Eindruck, dass Deutschland ein besseres System als die meisten anderen Länder hat. Außerdem zeichnete sich Deutschland in den letzten 30 Jahren durch ein außerordentlich hohes Maß an innenpolitischer Kontinuität aus. Ich bin 34 Jahre alt, und ich habe bislang nur drei deutsche Bundeskanzler erlebt. Ich erinnere mich sogar, dass ich einigermaßen verdutzt war, als die Kanzlerschaft von Helmut Kohl zu Ende ging: Er war fünf Jahre vor meiner Geburt an die Macht gekommen, und ihm folgte Gerhard Schröder nach, als ich elf war. Schröder regierte sieben Jahre. Und in den letzten 16 Jahren hat Angela Merkel als Bundeskanzlerin amtiert. Zum Vergleich: Ein gleichaltriger Amerikaner hat sieben Präsidentschaften erlebt. Ein Brite meines Alters hat sieben Premierminister bzw. Premierministerinnen gekannt und ein Italiener fast 20. Noch bemerkenswerter ist die Tatsache, dass Deutschland, abgesehen von sieben Jahren meines Lebens, eine Regierung hatte, die von derselben Partei geführt wurde - der CDU/CSU.

Diese internationale und innenpolitische Kontinuität hatte zur Folge, dass die Politik uns keinen definierenden Moment bot. Es gab keine Proteste wie 1968, um die wir uns hätten scharen können, kein 1989, in dem der Mauerfall gefeiert wurde, kein Krieg, der uns traumatisiert zurückließ (Gott sei Dank!), und keine Revolution, politische Revolte oder geopolitische Zeitenwende. Das eheste, was meine Generation als definierenden Moment vorweisen kann, ist die Fußballweltmeisterschaft von 2006, die von Deutschland ausgerichtet wurde. Zum ersten Mal erreichte uns die Geopolitik im Jahr 2015 in Form der Flüchtlingskrise. Aber 2015 waren selbst die jüngsten Millennials 20 Jahre alt, und die meisten waren 25 Jahre oder älter. Dies war zu spät (und auch nicht einschneidend genug), um unsere Sicht der Welt grundlegend zu verändern. Das Gleiche gilt für die gegenwärtige Pandemie.

Noch wichtiger ist, dass wir Kontinuität als den Normalzustand verinnerlichten. Auf einer emotionalen Ebene haben wir nie wirklich verstanden, dass sich die Lage ändern kann, und zwar recht schnell. Im Jahr 1989 war die Mauer plötzlich weg, und ein ganzes Regime, eine Lebensform verschwanden einfach. Die geopolitischen Fundamente gerieten ins Wanken. Dies muss sowohl aufregend als auch verwirrend gewesen sein. Wer dies miterlebte, erfuhr, dass Stabilität nicht garantiert ist. Meine Generation hat ein solches politisches Erdbeben nie erlebt. Die Fundamente sind heute stabil, also müssen sie immer stabil sein - wie könnte es anders sein? Und auch wenn wir auf einer intellektuellen Ebene wissen mögen, dass Stabilität nicht garantiert ist, ist es nicht das Gleiche. Es ist eine Sache, zu wissen, dass es Erdbeben gibt, eine ganze andere ist es, eines selbst zu erleben. Ich fürchte, dass wir nicht fähig sind, uns ein Erdbeben vorzustellen, ganz zu schweigen davon, uns auf eines vorzubereiten.

Selbstverständlich ist das Sprichwort „mögest du in interessanten Zeiten leben" als Fluch, nicht als Segen gedacht. Ereignisreiche Zeiten sind nur im Rückblick interessant, während es beunruhigend, niederdrückend und oftmals gefährlich ist, sie zu durchleben. Daher will ich mich nicht beklagen. Aber in ruhigen Zeiten zu leben, bringt seine eigenen Herausforderungen mit sich - insbesondere, wenn sich die Umstände ändern.

\section{„Das Ende der Geschichte ist für Sie bloß ein Konzept. Wir wurden hineingeboren, davon geprägt“"}

Francis Fukuyamas Idee vom „Ende der Geschichte“ wird oft in dem grob vereinfachenden Sinne von ,es wird keine bedeutenden Ereignisse mehr geben“ missverstanden. Aber auch, wenn sich diese grob vereinfachende Interpretation für Deutschland weitgehend als zutreffend erwies, sprach Fukuyama über Ideen, nicht Ereignisse. Er schrieb: „Womöglich sind wir nicht nur Zeugen des Endes des Kalten Krieges oder des Vorübergehens einer ungewöhnlichen Phase der Nachkriegsgeschichte, sondern des Endes der Geschichte als solche: das heißt des Endpunkts der ideologischen Entwicklung der Menschheit und der Universalisierung der westlich freiheitlichen Demokratie als der endgültigen Regierungsform. " Er behauptete, die freiheitliche Demokratie westlicher Prägung sei das einzige verbliebene Modell. Es zeigte sich, dass die Deutschen ihm nur allzu gern glauben wollten.

Vor drei Jahren schrieb Thomas Bagger einen ausgezeichneten Aufsatz über die Auswirkungen von 1989 auf die Einstellung der Deutschen. ${ }^{2}$ Er zeigte, dass die Deutschen die Idee vom Ende der Geschichte begeisterter aufnahmen als irgendjemand sonst, weil „gegen Ende eines Jahrhun-

2 https://www.atlantik-bruecke.org/the-world-according-to-ger many-reassessing-1989/. 
derts, das sich dadurch auszeichnete, dass es zweimal auf der falschen Seite der Geschichte gestanden hatte, Deutschland endlich auf der richtigen Seite stand." Die Staaten der Welt, so erklärten es Verfechter der Idee vom Ende der Geschichte, würden alle einem System zustreben, das (militärische) Macht entwertet und Probleme durch Regeln und Gesetze regelt. Regierungen würden Streitigkeiten im Rahmen internationaler Organisationen beilegen. Nationalismus und Ideologien würden ihre Anziehungskraft verlieren. Nach dem Dammbruch, den das Jahr 1989 darstellte, schienen diese Entwicklungen unausweichlich zu sein. All dies kam bei den Deutschen sehr gut an.

Der Primat des Rechts gegenüber der Macht war ein populäres Konzept für ein Land, das über sich selbst dachte, man könne ihm keine Macht anvertrauen. Der Liberalismus passt perfekt zu Deutschland, ebenso wie der Bedeutungsverlust der Persönlichkeit in der Politik. Der Bogen der Geschichte neigte sich der freiheitlichen Demokratie zu, so dass es auf die einzelnen Personen nicht mehr so sehr ankam. Man brauchte keinen „Führer“ mehr - ein Begriff, der in Deutschland zu Recht jegliche Legitimation verloren hat -, sondern Verwalter, Manager und Administratoren, die eine zwangsläufige Entwicklung beaufsichtigen und regelten. Dieser Ansatz mag zu erklären helfen, warum so viele deutsche Politikerinnen und Politiker eher langweilig sind. Eine der aufregendsten Nachrichten über Angela Merkel ist ihr Rezept für Kartoffelsuppe. In Deutschland ist es kein Manko, ein langweiliger Politiker zu sein - sondern ein Qualitätsmerkmal.

Bagger kommt zu dem Schluss, dass diese Erfahrung es Deutschen seiner Generation, die das Ende der Geschichte begeistert aufgenommen hatten, erschwere, sich an die veränderte geopolitische Lage in der Gegenwart anzupassen. Das mag sein, aber Bagger lässt die Tatsache unberücksichtigt, dass es eine Generation gibt, die noch stärker davon beeinflusst wurde als diejenigen, die 1989 selbst erlebt haben: die Generation, für die die Überzeugungen, die daraus hervorgingen, zur Norm wurden. Man mag heute die Naivität des post-1989 Optimismus belächeln. Aber wie können wir ihn aufgeben, wenn wir nie etwas Anderes gekannt haben? Die Generation vor uns hat den Geist von 1989 lediglich angenommen. Wir wurden in ihn hineingeboren und davon geprägt.

Lange Zeit schien die Realität unsere Überzeugungen zu stützen: Es ging uns gut, und immer mehr Menschen wollten so sein wie wir. Die gesamten 1990er Jahre hindurch - unsere Kindheit - hatte man in Westeuropa das Gefühl, auf der richtigen Seite zu stehen. Die Europäische Union expandierte rasch, da sich immer mehr Länder ihr anschließen wollten. Die Erwartung einer allgemeinen
Konvergenz sowie der Gedanke, die ganze Welt würde sich in Richtung Demokratie und Marktwirtschaft entwickeln und dass mit der Zeit alle so werden würden wie wir - diese Ideen wurden Teil unserer politischen DNA. Jeder, so glaubten wir, werde über kurz oder lang dem Beispiel Deutschlands nacheifern. Für uns ging es dabei nicht um eine ideologische Frage - wir hatten Ideologien und -ismen aller Art hinter uns gelassen und die Dinge waren jetzt so, wie sie sein sollten. Ideologische Auseinandersetzungen waren etwas für Geschichtsbücher, und wir sahen mit leichtem Mitleid auf diejenigen herab, die sich in der Vergangenheit in solche Kämpfe gestürzt hatten. Wir hatten uns auf eine höhere Ebene des Daseins begeben.

Wenn Sie Griechin oder Pole sind und diese Zeilen lesen, werden Sie aller Wahrscheinlichkeit nach diese Beschreibung des deutschen Denkens nicht bloß als arrogant, sondern auch als unzutreffend betrachten. Hat Deutschland in den letzten Jahren denn nicht eine Politik verfolgt, die in seinem Interesse war und nicht annähernd so aufgeklärt, wie es meine Beschreibung behauptet? Wie steht es mit Nord Stream 2? Was ist mit der europäischen Austeritätspolitik? Und hat Deutschland etwa nicht stärker als praktisch alle anderen Länder von der europäischen Integration und dem Euro profitiert? Ist all dieses Gerede über Werte und Freundschaft mehr als ein Deckmantel für die gute alte Interessenpolitik?

Ich persönlich glaube das nicht. Die Europäische Union ist gut für Deutschland, aber die EU stünde nicht da, wo sie heute steht, wenn Deutschland nicht gewillt gewesen wäre, Opfer $\mathrm{zu}$ bringen, die eigennützigere Länder nicht gebracht hätten, wie die Aufgabe der Deutschen Mark für den Euro. Nord Stream 2 ist meines Erachtens vor allem ein Beispiel für den Mangel an strategischem Denken in Deutschland: Wir Deutschen glaubten, wir hätten die Machtpolitik hinter uns gelassen und seien in eine Welt eingetreten, in der Wirtschaft am wichtigsten ist und der Handel alle zusammenbringt. Aber selbst wenn Sie anderer Meinung sind, ist das Narrativ das, was für deutsche Millennials zählt. Millennials beginnen gerade erst, Machtpositionen zu bekleiden. Wir sind mit dem Narrativ aufgewachsen, Machtpolitik sei etwas Schlechtes und sind davon überzeugt, dass wir das besser verstanden haben, als alle anderen.

\section{Eine gefährliche moralische Überlegenheit}

Wenn sich das für Sie arrogant anhört, dann sind Sie nicht allein. Mit der deutschen Ablehnung von Machtpolitik, 
Realpolitik und nationalen Interessen geht ein Gefühl moralischer Überlegenheit einher. Wir haben unsere Geschichte so gut aufgearbeitet, sind so weitentwickelt und aufgeklärt, dass wir den Nationalismus weitgehend hinter unter gelassen haben, dass wir uns nicht von Demagogen verführen lassen. Ja, wir haben es in der Vergangenheit gewaltig vermasselt, aber niemand hat die Lektionen der universellen Wahrheit besser gelernt als wir. Geopolitik, Interessenpolitik und Realpolitik sind daher Dinge, die wir weniger aufgeklärten Akteuren überlassen. Dieses Gefühl moralischer Überlegenheit ist nicht nur abstoßend und verärgert möglicherweise Verbündete, die nicht wie unaufgeklärte Verwandte behandelt werden wollen, es ist auch gefährlich, weil es unkritisch ist. Wir glaubten an die Schlagwörter von 1989, ohne zu erkennen, dass sie nur eine Lesart der Zukunft sind. Unserer Auffassung nach war die Konvergenz unvermeidlich - Chinas Mittelschicht würde nach Demokratie verlangen, sobald sie nur stark genug wäre, und Russlands Nationalismus würde langsam verblassen. Nicht zuletzt wegen dieser Überzeugungen traf uns der Wandel der internationalen Beziehungen in jüngster Zeit so gänzlich unvorbereitet. Wir konnten nicht nur nicht verstehen, was vor sich ging, es fiel uns auch schwer, unser System gegen Angriffe von außen und innen $z u$ verteidigen. Wenn man einfach weiß, dass ein vereintes Europa die Antwort ist, dass internationale Zusammenarbeit notwendig ist, dass die Herrschaft des Rechts besser ist als Machtpolitik und dass all dies einfach richtig ist, dann kann es erstaunlich schwer sein, dies jemandem zu erklären, der diese Prämisse in Frage stellt.

Die moralische Überlegenheit ignoriert auch folgendes: Während wir uns von - aus unserer Sicht - altmodischen Ideen wie militärischer Macht verabschiedet haben, spannen andere - die NATO und die Vereinigten Staaten einen militärischen Schutzschirm über uns auf, der uns den Luxus erlaubt, militärische Macht abzuwerten. Und das Ende der Geschichte nahm uns unsere Zukunft. Schließlich wussten wir, wo der Prozess enden würde. Die Politik wurde langweilig - ein Verwaltungsakt statt einer Arena des ideologischen Wettstreits. Dies erklärt vielleicht auch, warum alle deutschen Parteien zwangsläufig die politische „Mitte“ beanspruchen. Es scheint nicht notwendig zu sein, strategisch über die Zukunft nachzudenken.

\section{Fazit}

Ich beklage mich nicht darüber, dass meine Generation eine großartige Kindheit hatte - stabil, sicher und von der Erwartung erfüllt, die Zukunft würde noch besser werden. Aber wir wuchsen in einer außergewöhnlichen Welt auf, die wir als den Normalzustand ansahen. Nun, da sich die internationale Politik ändert, sind wir ratlos. Natürlich mag ich mich irren. Jemand nannte mich einmal „den ältesten jungen Mensch,“ den er kenne, was ich als Kompliment auffasste, auch wenn es vermutlich nicht so gemeint war. Also bin ich vielleicht diejenige, die das Licht nicht sieht und nicht versteht, dass sich die Welt tatsächlich verändert hat. Aber es beunruhigt mich, wenn in Konfliktsimulationen mit anderen Millennials niemand intuitiv auf eine Situation reagiert, indem er sich erst einmal seinen eigenen Interessen und Fähigkeiten und die seiner Gegenspieler vergegenwärtigt, und eine Strategie formuliert, die beiden gerecht wird. Es besorgt mich, dass wir in einem Moment, in dem die internationale Ordnung zerbrechlich ist und von Akteuren, die nicht unser Bestes wollen, Alternativen aufgebracht werden, so unfähig zu strategischem Denken zu sein scheinen. Ich bezweifle, dass wir auf die nächste Generation deutscher außenpolitischer Denker und Macher zählen können. Wir haben eine Generation von Deutschen, die die Dinge als selbstverständlich betrachtet haben und denen es schwerfällt, Antworten auf Herausforderungen zu finden. Meine Generation hofft insgeheim, dass wir bald zu unserem Normalzustand zurückkehren werden und dass wir diese unaufgeklärte Machtpolitik hinter uns lassen, um uns den echten Herausforderungen wie dem Klimawandel zu stellen. Aber die Welt dürfte uns kaum diesen Gefallen machen. Um dieser Herausforderung gewachsen zu sein, muss meine Generation ihren strategischen Muskel trainieren - und zwar schnell. 